\title{
Nonlinear dynamics modelling of multistage micro-planetary gear transmission
}

\author{
Jianying $\mathrm{Li}^{1}$, Zhiyong $\mathrm{Xu}^{2}$, Qingchun $\mathrm{Hu}^{3}$, Changfu Zong ${ }^{1}$ and Tianjun $\mathrm{Zhu}^{1}$ \\ ${ }^{1}$ School of Mechanical and Automotive Engineering, ZhaoQing University, Zhaoqing 516260, Guangdong, China, \\ ${ }^{2}$ School of Electronics and Electrical Engineering, ZhaoQing University, Zhaoqing 516260, Guangdong, China, \\ ${ }^{3}$ School of Mechanical and Automotive Engineering, South China University of Technology, Guangzhou510640, China
}

\begin{abstract}
The transmission structure of a $2 \mathrm{~K}-\mathrm{H}$ multistage micro-planetary gear transmission reducer is described in detail, and three assumptions are supposed in dynamic modelling. On basis of these assumptions, a three stages $2 \mathrm{~K}-\mathrm{H}$ micro-planetary gear transmission dynamic model is established, in which the relative displacement each meshing gear pairs can be obtained after including the comprehensive transmission error. According to gear kinematics, the friction arms between the sun gear, the ring gear and the $n^{\text {th }}$ planet are also obtained, and the friction coefficient in the mixed elastohydrodynamic lubrication is considered, the transmission system motion differential equations are obtained, including above factors and the time-varying meshing stiffness, damping and backlash, inter-stage coupling stiffness, it can be provided an theoretical foundation for further analysing the parameter sensitivity, dynamic stability and designing.
\end{abstract}

\section{Introduction}

The micro-planetary gear transmission is widely used in aviation equipment, space technology and medical equipment, small cars and so on [1], because it has the characteristics of planetary transmission and miniature gear. The appearance background, structure, size and function of micro-planetary gear reducer were discussed in the literature [2]. The design calculation and the modular design of micro-planetary gear transmission were discussed in detail $[3,4]$. The manufacturing and intelligent assembly processes of micro-planetary gear reducer were analyzed after briefly introducing its advantage and application prospect [5]. The strength of the tubular multistage micro-planetary gear reducer was analyzed in Ref. [6]. The lubrication is a very important problem during the development of micro-planetary gear transmission[7], concerning about the friction field and establishing the nonlinear dynamic model of multistage micro-planetary gear have not been reported, but Zhu EnYong, et al. [8]built the translational-torsional nonlinear dynamic model including the friction, timevarying meshing stiffness, backlash and comprehensive transmission error. Wang Haofei[9] studied the dynamic properties of double helical planetary gear based on the tooth surface friction. Chen Changzheng, et al [10] established multi-degree of freedom translationaltorsional nonlinear dynamic model with mixed elastohydrodynamic lubrication friction, time-varying meshing stiffness, and transmission error, backlash. Bao
Heyun, et al. [11] established a translational-torsional dynamic model with the friction, time-varying meshing stiffness and damping, comprehensive transmission error and flexible deformable ring gear beyond pitch point meshing. Chen $\mathrm{Fu}$, et al. [12] built two-stage planetary gearbox coupling torsion dynamic model considering the tooth surface friction, time-varying meshing stiffness and inter-stage coupling stiffness.

The non-linear dynamic model of multistage microplanetary transmission including the tooth surface friction, time-varying meshing stiffness, comprehensive transmission error, backlash and inter-stage coupling stiffness in this paper, it can be provided a theoretical foundation for further dynamic analysis.

\section{Dynamic modelling of multistage micro-planetary gear transmission}

\subsection{Dynamic model of multistage micro- planetary gear transmission}

The transmission diagram of a $2 \mathrm{~K}-\mathrm{H}$ multistage microplanetary gear reducer is shown in Fig. 1. The power inputs through the first stage sun gear $\left(\mathrm{s}^{\mathrm{I}}\right)$, the third stage carrier is output $\left(\mathrm{c}^{\mathrm{III}}\right)$. The connections of the former stage carrier and the rear stage sun gear are regarded as the spring and damping, the connections of the former stage ring gear $\left(\mathrm{r}^{i}\right)$ and the rear stage ring gear are also regarded as spring and damping, and each stage ring gear connects with the body of a $2 \mathrm{~K}-\mathrm{H}$ multistage micro-planetary gear 
reducer.

The following assumptions are supposed in dynamic modelling:

(1) All gears are involute spur gears and the tooth meshing force is always in the meshing plane;

(2) The same stage planetary gears distribute evenly with the same quality, size and moment of inertia;

(3) The bearing stiffness of each stage sun gear, carrier and planetary gears are linear stiffness.

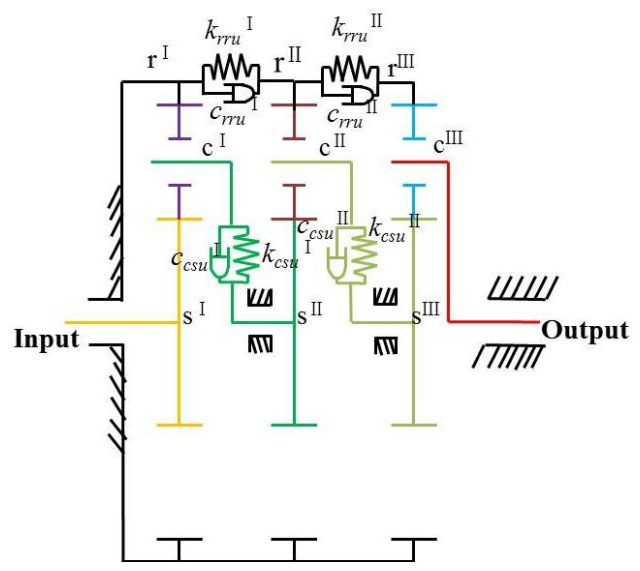

Figure 1. The transmission diagram of a $2 \mathrm{~K}-\mathrm{H}$ multistage micro-planetary gear reducer

Based on the above assumptions, the co-moving coordinates of each stage carrier are modeled. Unless specially indicating, $i=\mathrm{I}$, II ,III represent planet set I , II and III , respectively. $n=1,2, \cdots N_{i}$, where $N_{\mathrm{I}}, N_{\mathrm{II}}, N_{\mathrm{III}}$ represent numbers of planetary gear of planet set I II and III. The three stage micro-planetary gear transmission dynamic model is established through inter-stage coupled method based on the translationaltorsional dynamic model (See Fig. 2). $x_{h}^{i}, y_{h}^{i}, u_{h}^{i},(h=s, c, r)$ are translational and torsional displacements of component $h$ of planet set $i$ (the sun gear, carrier and ring gear), respectively. $\theta_{h}^{i}$ is angular displacement of component $h$ of planet set $i$, and $u_{h}^{i}=r_{h}^{i} \theta_{h}^{i}$, where $r_{h}^{i}$ is base radius of component $h$ of planet set $i\left(r_{c}^{i}\right.$ is radius of the circle passing through planetary gear centers). $x_{n}^{i}, y_{n}^{i}, u_{n}^{i}$ are translational and torsional displacements of the $n^{\text {th }}$ planet of planet set $i$, respectively. $m_{h}^{i}, I_{h}^{i}$ are mass and moment of inertia of component $h$ of planet set $i ; m_{n}^{i}, I_{n}^{i}$ are mass and moment of inertia of the $n^{\text {th }}$ planet of planet set $i ; k_{h j}^{i}, c_{h j}^{i}(j=x, y, u)$ are bearing stiffness and damping, torsional stiffness and damping of component $h$ of planet set $i, k_{h^{\prime} n}^{i}, c_{h^{\prime} n}^{i}\left(h^{\prime}=\mathrm{s}, \mathrm{r}\right)$ are meshing stiffness and damping between the sun gear, ring gear and the $n^{\text {th }}$ planet of planet set $i ; k_{\mathrm{p} n}^{i}, c_{\mathrm{p} n}^{i}$ are bearing stiffness and damping of the $n^{\text {th }}$ planet of planet set $i ; k_{r r u}^{i}, c_{r r u}^{i}$ are coupling torsional stiffness and damping of the former stage ring gear and the rear stage ring gear of planet set $i$; $k_{\mathrm{csu}}^{i}, c_{\mathrm{csu}}^{i}$ are coupling torsional stiffness and damping of the former stage carrier and the rear stage sun gear. $b_{h^{\prime} n}^{i}$ is the backlash between the sun gear, ring gear and the $n^{\text {th }}$ planet of planet set $i$.

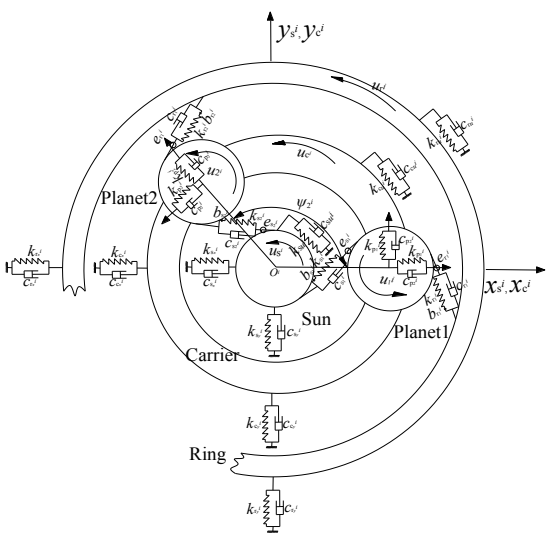

Figure 2. Dynamic model of a $2 \mathrm{~K}-\mathrm{H}$ multistage microplanetary gear transmission system

\subsection{Relative displacement and meshing force}

The relative positional relationship of each meshing gear pairs of a $2 \mathrm{~K}-\mathrm{H}$ multistage micro-planetary gear transmission can be obtained after including the comprehensive transmission error.

1) The elastic deformation between the sun gear and the $n^{\text {th }}$ planet of planet set $i$

$\delta_{\mathrm{s} n}^{i}(t)=\left(x_{n}^{i}-x_{\mathrm{s}}^{i}\right) \sin \psi_{\mathrm{s} n}^{i}+\left(y_{\mathrm{s}}^{i}-y_{n}^{i}\right) \cos \psi_{\mathrm{s} n}^{i}+u_{\mathrm{s}}^{i}+u_{n}^{i}+e_{s n}^{i}(t)$

2) The elastic deformation between the ring gear and the $n^{\text {th }}$ planet of planet set $i$

$$
\delta_{\mathrm{rn}}^{i}(t)=\left(x_{n}^{i}-x_{\mathrm{r}}^{i}\right) \sin \psi_{\mathrm{r} n}^{i}+\left(y_{\mathrm{r}}^{i}-y_{n}^{i}\right) \cos \psi_{\mathrm{r} n}^{i}+u_{\mathrm{r}}^{i}-u_{n}^{i}+e_{r n}^{i}(t)
$$

3) The linear displacement and tangential direction elastic deformation of the carrier relative to the $n^{\text {th }}$ planet of planet set $i$

$$
\begin{gathered}
\delta_{c n x}^{i}(t)=x_{\mathrm{c}}^{i}-x_{n}^{i}-u_{c}^{i} \sin \psi_{n}^{i} \\
\delta_{c n y}^{i}(t)=y_{\mathrm{c}}^{i}-y_{n}^{i}+u_{\mathrm{c}}^{i} \cos \psi_{n}^{i} \\
\delta_{c n u}^{i}(t)=\left(x_{n}^{i}-x_{c}^{i}\right) \sin \psi_{n}^{i}+\left(y_{c}^{i}-y_{n}^{i}\right) \cos \psi_{n}^{i}+u_{c}^{i}
\end{gathered}
$$

where $\psi_{n}^{i}(t)$ is the $n^{\text {th }}$ planet location of planet set $i$ with $x$ axis positive direction. $\psi_{n}^{i}(t)=\omega_{c}^{i} t+2 \pi(n-1) / N_{i} \cdot \psi_{m}^{i}(t)=\psi_{n}^{i}(t)+\alpha_{r}^{i}, \quad \psi_{s n}^{i}(t)=\psi_{n}^{i}(t)-\alpha_{s}^{i}$, where $\alpha_{r}^{i}, \alpha_{s}^{i}$ is pressure angle of the ring gear and sun gear of planet set $i$, respectively. $e_{h^{\prime} n}^{i}$ is the comprehensive transmission error between the sun gear, ring gear and the $n^{\text {th }}$ planet of planet set $i[13]$.

$$
\begin{gathered}
e_{s n}^{i}(t)=E_{s n}^{i} \sin \left[\omega_{m}^{i}\left(t+\gamma_{s n}^{i} T_{m}^{i}\right)\right] \\
e_{r n}^{i}(t)=E_{r n}^{i} \sin \left\{\omega_{m}^{i}\left[t+\left(\gamma_{r n}^{i}+\gamma_{s r}^{i}\right) T_{m}^{i}\right]\right\}
\end{gathered}
$$

where comprehensive transmission error amplitude between the sun gear, ring gear and the $n^{\text {th }}$ planet of planet set $i E_{s n}^{i}, E_{r n}^{i}$; meshing frequency and period $\omega_{m}^{i}, T_{m}^{i}$; internal and external meshing phasing $\gamma_{r n}^{i}, \gamma_{s n}^{i}$; phasing between internal and external meshing for a given 
planet $\gamma_{s r}^{i}$.

The backlash can be described by piecewise function between the sun gear, ring gear and the $n^{\text {th }}$ planet of planet set $i$

$$
\delta_{h^{\prime} n}^{b}(t)=\left\{\begin{array}{cc}
\delta_{h^{\prime} n}^{i}(t)-b_{h^{\prime} n}^{i}, & \delta_{h^{\prime} n}^{i}(t) \geqslant b_{h^{\prime} n}^{i} \\
0, & \left|\delta_{h^{\prime} n}^{i}(t)\right|<b_{h^{\prime} n}^{i} \\
\delta_{h^{\prime} n}^{i}(t)+b_{h^{\prime} n}^{i}, \delta_{h^{\prime} n}^{i}(t) \leqslant-b_{h^{\prime} n}^{i}
\end{array}\right.
$$

The time-varying meshing stiffness with the $n^{\text {th }}$ planet of planet set $i k_{h^{\prime} n}^{i}(t)$

$$
k_{h^{\prime} n}^{i}(t)=\bar{k}_{h^{\prime} n}^{i}+\sum_{l=1}^{\infty}\left(a_{h^{\prime} n}^{i(l)} \sin l \omega_{\mathrm{m}}^{i} t+b_{h^{\prime} n}^{i(l)} \cos l \omega_{\mathrm{m}}^{i} t\right)
$$

$\bar{k}_{h^{\prime} n}^{i}$ is average meshing stiffness with the $n^{\text {th }}$ planet of planet set $i ; a_{h^{\prime} n}^{i(l)}, b_{h^{\prime} n}^{i(l)}$ are Fourier series expansion coefficient; $l$ is Fourier series expansion number.

The time-varying meshing force of a $2 \mathrm{~K}-\mathrm{H}$ multistage micro-planetary gear transmission can be expressed as

$$
F_{m h^{\prime} n}^{i}(t)=k_{h^{\prime} n}^{i}(t) \delta_{h^{\prime} n}^{b}(t)+c_{h^{\prime} n}^{i}(t) \dot{\delta}_{h^{\prime} n}^{b}(t)
$$

\subsection{Friction arm}

The diagram of the outer and internal meshing gear pairs friction arm are shown in Fig. 3, $p^{i}$ is pitch point in the outer meshing gear pairs friction arm diagram (Seen Fig. 3(a)), $N_{1}^{i} N_{2}^{i}$ is theoretical line of action, $B_{1}^{i} B_{2}^{i}$ is actual line of action. $r_{b}^{i}, r_{a}^{i}$ are radius of the gear base circle and addendum circle, $p_{b t}^{i}$ is gear normal pitch. The symbolic meaning shown in Fig. 3(b) is similar to Fig. 3 (a).

According to gear kinematics, the friction arm between the sun gear and the $n^{\text {th }}$ planet of planet set $i[8,12]$

$$
\begin{gathered}
l_{\mathrm{s} n, k}^{i}=\left(r_{b s}^{i}+r_{b n}^{i}\right) \tan \alpha_{s n}^{i}-\sqrt{\left(r_{a n}^{i}\right)^{2}-\left(r_{b n}^{i}\right)^{2}}+(k-1) p_{b t}^{i}+M_{k}^{i}(11) \\
l_{n s, k}^{i}=\sqrt{\left(r_{a n}^{i}\right)^{2}-\left(r_{b n}^{i}\right)^{2}}-(k-1) p_{b t}^{i}-M_{k}^{i}
\end{gathered}
$$

Similarly, the friction arm between the ring gear and the $n^{\text {th }}$ planet of planet set $i[8,12]$

$$
\begin{gathered}
l_{r n, k}^{i}=\sqrt{\left(r_{a r}^{i}\right)^{2}-\left(r_{b r}^{i}\right)^{2}}+(k-1) p_{b t}^{i}+M_{k}^{i} \\
l_{n r, k}^{i}=\sqrt{\left(r_{a r}^{i}\right)^{2}-\left(r_{b r}^{i}\right)^{2}}-\left(r_{b r}^{i}-r_{b n}^{i}\right) \tan \alpha_{r n}^{i}+(k-1) p_{b t}^{i}+M_{k}^{i}
\end{gathered}
$$

where $M_{k}^{i}=\bmod \left(\omega_{n}^{i} r_{b b}^{i}, p_{b t}^{i}\right)$, and "mod" is the modulus function defined as $\bmod (x, y)=x-y \cdot \operatorname{floor}(x / y)$, if $y \neq 0 . \omega_{n}^{i}$ is gear angular velocity of planet set $i$.

\subsection{Friction coefficient}

The lubrication of a $2 \mathrm{~K}-\mathrm{H}$ multistage micro-planetary gear transmission is regarded as in the mixed elastohydrodynamic lubrication and its friction coefficient of meshing gear pairs of planet set $i[14,15]$

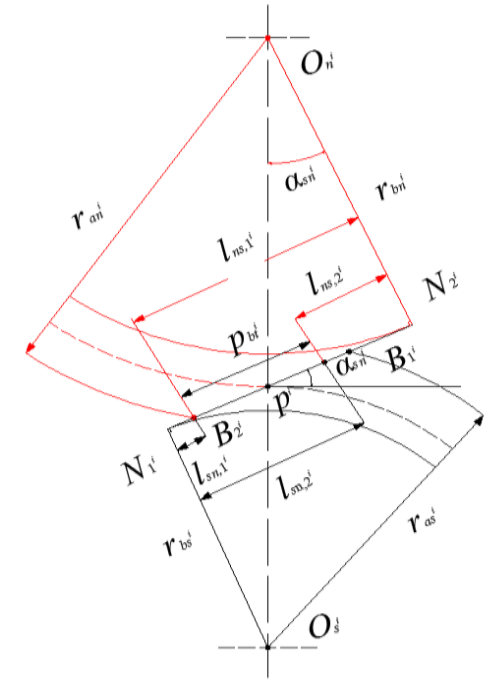

(a) Outer mesh gear pairs

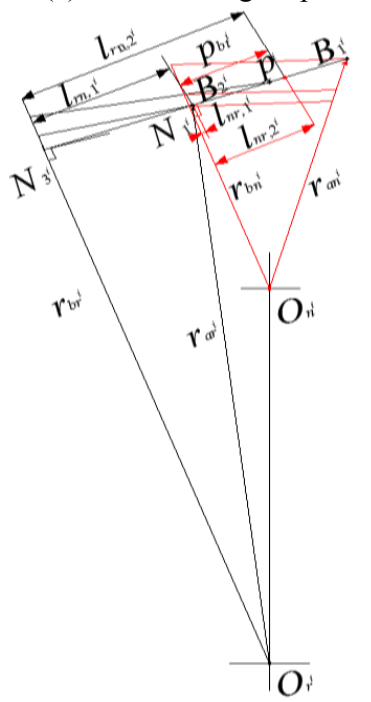

(b) Internal mesh gear pairs

Figure 3. The diagram of the outer and internal meshing gear pairs friction arm

$\mu^{i}=\left[0.0127 \zeta^{1.2}\left(\frac{50}{50-39.37 \sigma}\right) \lg \frac{29.66 \mathrm{~W}}{\rho V_{s}(\varphi) V_{R}^{2}(\varphi)}+0.15(1-\zeta)\right]^{i}$

where $\rho$ is viscosity of the lubricating oil; $V_{s}(\varphi), V_{R}(\varphi)$ are instantaneous sliding and rolling speed of the meshing point, respectively, as shown in the equations (16) and (17)

$V_{s}(\varphi)=\frac{\pi}{30} n_{1} r_{b 1}\left[\left(1+\frac{1}{\kappa}\right) \tan \alpha^{\prime}-\left(1+\frac{1}{\kappa}\right) \tan \alpha\right] \times 10^{-3}(16)$

$V_{R}(\varphi)=\frac{\pi}{30} n_{1} r_{b 1}\left[\left(1-\frac{1}{\kappa}\right) \tan \alpha^{\prime}+\left(1+\frac{1}{k}\right) \tan \alpha\right] \times 10^{-3}(17)$

where $n_{1}$ is driving gear speed; $r_{b 1}$ is base radius of the driving gear; $\kappa$ is transmission ratio; $\alpha^{\prime}$ is pressure angle of the circle of the meshing point; $\alpha$ is pressure angle of the pitch circle.

$\zeta$ shown in the equation (15) is bearing ratio of full film lubrication in mixed lubrication, so the bearing ratio 
of the meshing gear pairs full film lubrication area is shown in the equation (18)[16]

$$
\zeta^{i}=\frac{1.21\left(h_{\min }^{i} / \sqrt{\sigma_{1}^{i^{2}+\sigma_{2}^{i^{2}}}}\right)^{i 0.64}}{1+0.37\left(h_{\min }^{i} / \sqrt{\left.\sigma_{1}^{i^{2}+\sigma_{2}^{i^{2}}}\right) i^{1.26}}\right.}
$$

where $h_{\min }^{i}$ is minimum oil film thickness; $\sigma_{1}^{i}, \sigma_{2}^{i}$ are rootmean-square deviation of the profile of meshing tooth faces of the driving and driven gear, respectively.

The minimum oil film thickness can be calculated by the equation (19)[17]

$$
h_{\min }^{i}=\left[\frac{2.65 \alpha^{0.54}\left(\eta_{0} U\right)^{0.7} R^{0.43}}{E^{\prime 0.03} W^{0.13}}\right]^{i}
$$

where $\alpha$ is lubricating oil viscosity coefficient; $\eta_{0}$ is dynamic viscosity of the lubricating oil at normal temperature; $U$ is average speed of tooth faces; $R$ is radius of the equivalent curvature of the meshing tooth; $E^{\prime}$ is comprehensive elastic modulus of materials, $W$ is unit normal load of the meshing tooth. They are shown in the equations (20)-(23).

$$
\begin{gathered}
U=\frac{\pi}{60} n_{1} r_{b 1}\left[\left(1-\frac{1}{\kappa}\right) \tan \alpha^{\prime}+\left(1+\frac{1}{\kappa}\right) \tan \alpha\right] \\
E^{\prime}=2\left[\left(1-v_{1}^{2}\right) / E_{1}+\left(1-v_{2}^{2}\right) / E_{2}\right]^{-1} \\
R=r_{b 1} \tan \alpha^{\prime}-\frac{r_{b 1} \tan ^{2} \alpha^{\prime}}{(1+\kappa) \tan \alpha} \\
W=\left\{\begin{array}{l}
\frac{F_{n}}{2 b} \text { Double teeth mesh } \\
\frac{F_{n}}{b} \text { Single tooth mesh }
\end{array}\right.
\end{gathered}
$$

where $v_{1}, v_{2}$ are Poisson's ratio of the driving and driven gear, respectively; $E_{1}, E_{2}$ are elastic modulus of the driving and driven gear; $F_{n}$ is normal force; $b$ is tooth width.

The meshing tooth friction force of the mixed elastohydrodynamic lubrication

$$
F_{f h^{\prime} n}^{i}(t)=\lambda^{i} \mu^{i} F_{m h^{\prime} n}^{i}(t)
$$

where $\lambda^{i}$ is direction coefficient of the friction force, it changes with the relatively rotation speed of the driving and driven gear, and can be taken as $1,0,-1$.

\section{Motion differential equations of multistage transmission}

According to Newton's second law, the lumped parameter dynamic model of a $2 \mathrm{~K}-\mathrm{H}$ multistage micro-planetary gear transmission system is established, so its motion differential equations of the ring gear, sun gear, carrier and and the $n^{\text {th }}$ planet of planet set $i$ are shown in the equations (25)-(28).

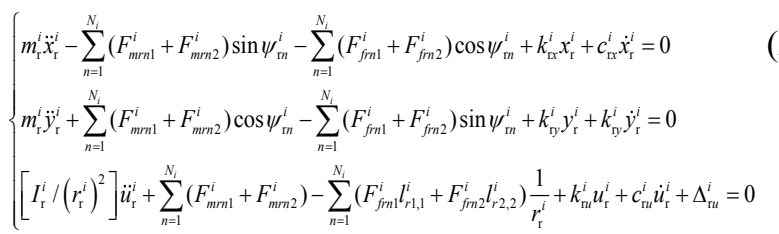

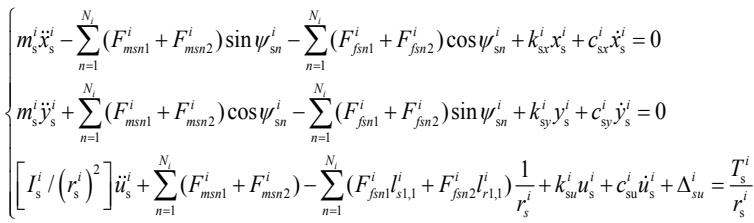

$\left\{\begin{array}{l}m_{\mathrm{c}}^{i} \ddot{x}_{\mathrm{c}}^{i}+\sum_{n=1}^{N_{i}}\left(k_{\mathrm{p} n}^{i} \delta_{c n x}^{i}+c_{\mathrm{p} n}^{i} \dot{\delta}_{c n x}^{i}\right)+k_{\mathrm{cx}}^{i} x_{\mathrm{c}}^{i}+c_{\mathrm{cx}}^{i} \dot{x}_{\mathrm{c}}^{i}=0 \\ m_{\mathrm{c}}^{i} \ddot{y}_{\mathrm{c}}^{i}+\sum_{n=1}^{N_{i}}\left(k_{\mathrm{p} n}^{i} \delta_{c n y}^{i}+c_{\mathrm{p} n}^{i} \dot{\delta}_{c n y}^{i}\right)+k_{\mathrm{cy}}^{i} y_{\mathrm{c}}^{i}+c_{\mathrm{cy}}^{i} \dot{y}_{\mathrm{c}}^{i}=0 \\ {\left[I_{\mathrm{c}}^{i} /\left(r_{\mathrm{c}}^{i}\right)^{2}\right] \ddot{u}_{\mathrm{c}}^{i}+\sum_{n=1}^{N_{i}}\left(k_{\mathrm{p} n}^{i} \delta_{c n u}^{i}+c_{\mathrm{p} n}^{i} \dot{\delta}_{c n u}^{i}\right)+k_{\mathrm{c} u}^{i} u_{\mathrm{c}}^{i}+c_{\mathrm{cu}}^{i} \dot{u}_{\mathrm{c}}^{i}+\Delta_{c u}^{i}=-\frac{T_{\mathrm{c}}^{i}}{r_{\mathrm{c}}^{i}}}\end{array}\right.$

$m_{n}^{i} r_{n}^{i}+\left(F_{m m 1}^{i}+F_{m m 2}^{i}\right) \sin \psi_{\mathrm{m}}^{i}+\left(F_{f m 1}^{i}+F_{f m 2}^{i}\right) \cos \psi_{\mathrm{m}}^{i}$

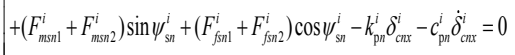

$\left\{m_{n}^{i} \dot{y}_{n}^{i}-\left(F_{m m 1}^{i}+F_{m m 2}^{i}\right) \cos \psi_{\mathrm{m} n}^{i}+\left(F_{f m 1}^{i}+F_{f m 2}^{i}\right) \sin \psi_{\mathrm{m}}^{i}\right.$

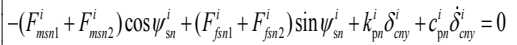

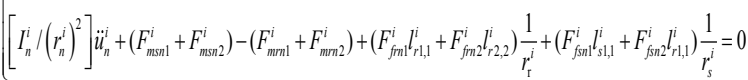

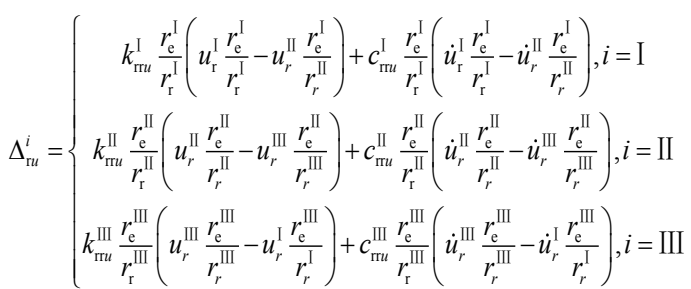

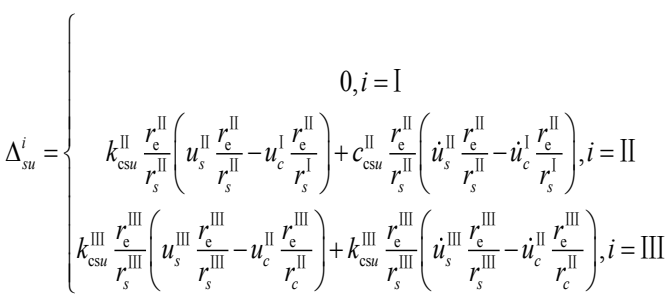

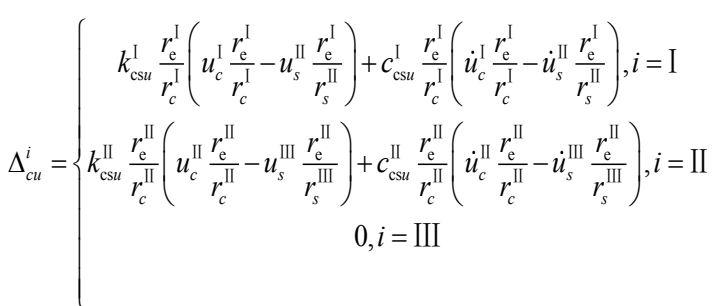

where $\Delta_{j u}^{i}$ is torsional coupling term of the component $h$ 
of planet set $i$.

\section{Conclusions}

A three stages $2 \mathrm{~K}-\mathrm{H}$ micro-planetary gear transmission mechanism is taken as the research object in this paper, on the basis of modeling assumptions, the lumped parameter dynamic model of a $2 \mathrm{~K}-\mathrm{H}$ multistage microplanetary gear transmission system is established and its motion differential equations are obtained, including the time-varying meshing stiffness, damping, transmission error and backlash, inter-stage coupling stiffness, and considering tooth face friction and lubrication condition of the meshing gear pairs, it can be provided an theoretical foundation for further analyzing the parameter sensitivity, dynamic stability and dynamic designing.

\section{References}

1. Z.G. Rao, Drive System Technique 27,16-22 (2013) (in Chinese)

2. Z.G. Rao, J.S. Maehine Building \& Automation 1011,15 (1999) (in Chinese)

3. Z.G. Rao, Drive System Technique 18-24,34 (2003) (in Chinese)

4. Z.G. Rao, Drive System Technique 26,10-17 (2012) (in Chinese)

5. Y.Z. Zhao, Machinery Design \& Manufacture 242243(2010)(in Chinese)
6. C.P.Yang, HeFei University of Technology (HeFei, 2012) (in Chinese)

7. Z.G Rao, Chemical Industry Press (Beijing, 2014) (in Chinese)

8. E.Y. Zhu, S. J. Wu, X.S. Wang, M.X. Deng, B. Qian, J. Vibration and shock 29, 217 -221(2010) (in Chinese)

9. H.F. Wang, Nanjing University of Aeronautics and Astronautics (Nanjing, 2015) (in Chinese)

10. C.Z. Chen, L. Zhang, J. Liu, Z.Q. Sun, X.T. Bai, Acta Energiae Solaris Sinica 37, 1695-1701(2016) (in Chinese)

11. H.Y. Bao, X.J. Zhou, R.P. Zhu, F.X. Lu, J. of Central South University (Science and Technology) 47, 3005-3010 (2016) (in Chinese)

12. F. Chen, Z.M. Xiao, L.R. Huan, Machine Design and Research 33, 63-71 (2017) (in Chinese)

13. C. Zhang, Higher Education Press (Beijing, 2008) (in Chinese)

14. J. Castro, J. Seabra, Proceedings of the Institution of Mechanical Engineers, Part J: Journal of Engineering Tribology (2007)

15. B. Wang, X.B. Chen, J. Tongji University (Natural Science), 42, 1904-1911 (2014) (in Chinese)

16. Y.Z. Hu, D. Zhu, J. Tribology 122, 1-9 (2000) (in Chinese)

17. N.X. Wang, X.P. Jiang, J. Shaanxi University of Science \& Technology 27, 84-86 (2009) (in Chinese) 